\title{
Tunneling Induced Dephasing And Pauli Blocking In InP Quantum Dots
}

\author{
Yasuaki Masumoto $^{\mathrm{a}}$, Fumitaka Suto ${ }^{\mathrm{a}}$, Michio Ikezawa ${ }^{\mathrm{a}}$, Chikako Uchiyama $^{\mathrm{b}}$ \\ and Masaki Aihara ${ }^{\mathrm{c}}$ \\ a'Institute of Physics, University of Tsukuba, Tsukuba, 305-8571 Japan \\ ${ }^{\mathrm{b}}$ Faculty of Engineering, Yamanashi University, Kofu, Yamanashi 400-8511, Japan \\ ${ }^{\circ}$ Graduate School of Material Science, Nara Institute of Science and Technology, Ikoma, Nara 630-0101, Japan
}

\begin{abstract}
We observed the tunneling induced dephasing and Pauli blocking in InP quantum dots (QDs). A highlysensitive heterodyne-detected photon echo method enabled us to observe the signal from one layer of self-assembled InP QDs in charged or neutral condition controlled by the electric field. The photon echo signal decreased much with the increase of electron doping. The photon echo of neutral InP QDs under the electric field showed tunneling-induced dephasing, which decays non-exponentially reflecting the non-Markovian nature of the tunneling process.
\end{abstract}

Tunneling process is a key issue in quantum structures. In the presence of tunneling from a discrete level in the quantum structure to a continuous state outside of it, the coupling between the electron wave functions in and out of the quantum structure causes the dephasing and relaxation. Dynamical process of tunneling from the quantum levels to the continuum state observed in the time domain so far has been discussed only quasi-classically by means of WKB approximation. Quantum mechanical process of tunneling from quantum dots (QDs) has never been observed directly in the time domain. Coherent nature of charged as well as neutral QDs is very important in coherent optical processing but is unknown because of lack of the experimental data. We developed a highlysensitive heterodyne-detected photon echo technique and successfully observed the tunneling-induced dephasing in one layer of neutral InP self-assembled QDs (base diameter $=40 \mathrm{~nm}$; height $=5 \mathrm{~nm}$ ) grown on an $\mathrm{n}^{+}$-GaAs substrate with an areal density of $10^{10} \mathrm{~cm}^{-2}$. InP QDs in our samples are non-intentionally n-doped and that dots can be neutralized under the negative electric bias $[1,2]$. We also observed that the charged dots do not produce the photon echo signal. Although the photon echo is known as a powerful tool to study the coherent nature of photo-excitation, it has not been used to study one layer of self-assembled QDs under the electric field so far.
The photon echo signal from the InP QDs showed two dramatic changes depending on the electric bias. When the electric bias is changed from $0 \mathrm{~V}$ to $-1.0 \mathrm{~V}$, the InP QDs are changed to be neutral and the photon echo signal increases by 4 orders of magnitudes and is at the maximum, while luminescence intensity is reduced. Coherently excited excitons in neutral dots act as electric dipoles producing the photon echo, while excitons in the QDs show non-radiative decay due to ionization and tunneling of holes under the negative electric bias. Around $0.1 \mathrm{~V}, \operatorname{InP} \mathrm{QDs}$ contain 2 excess electrons which prohibit the formation of the optical dipole due to the Pauli blocking and hence reduce the photon echo signal of the exciton.

The photon echo signal is not described by a single exponential decay but a Gaussian-like decay. When the negative electric bias is increased further from -1.0 $\mathrm{V}$ to $-2.5 \mathrm{~V}$, the hole tunneling is induced and the luminescence of InP QDs is quenched [3,4]. The decay rate of the photon echo evaluated at the initial part drastically increases and it can be fitted by exp($\left.U_{0}|U|\right)$, where $U_{0}=8 \mathrm{~V}$ is a fitting parameter and $U$ is the electric bias. The electric bias dependence of the dephasing rate and that of half of the luminescence decay rate well meet that of the WKB tunneling rate of the hole from the QDs, which assures the successful direct observation of hole tunneling from QDs. 

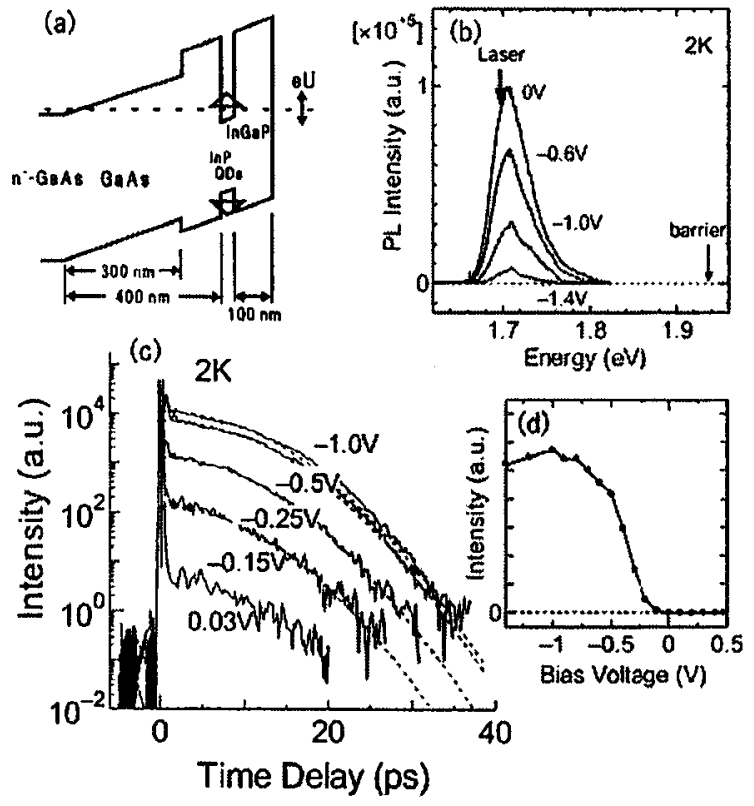

FIGURE 1. (a) Schematic picture of the potential profile. (b) Photoluminescence spectrum of InP QDs under the selective excitation of the dots at $1.85 \mathrm{eV}$ below the $\mathrm{In}_{0.5} \mathrm{Ga}_{0.5} \mathrm{P}$ barrier band gap. The energy of the 80 fs laser pulses for the photon echo experiments is shown. (c) Photon echo intensity as a function of electric voltage ranging from $0.03 \mathrm{~V}$ to $-1.0 \mathrm{~V}$. Dashed lines are the fittings by the nonMarkovian theory. (d) The echo intensity at 1 ps as a function of electric voltage.

We note that the decay of the photon echo signal is not exponential. The echo intensity slowly decays in short times, and tends to the exponential decay at later times. To analyze the echo decay, we used a model that a discrete state inside a dot is coupled with the continuous states outside the dot. Because of the coupling arising from the hole tunneling, the hole generated by the light pulse leaks out of dots. This model generally describes the non-Markovian dephasing, and the formula for the echo intensity is obtained as,

$$
\begin{gathered}
I(t)=\operatorname{Exp}\left(-2 \mathrm{~S}\left(\mathrm{t} /-\tau_{s}\right)-2 \mathrm{~S}\left(\tau_{s}\right)-\Delta_{i}^{2}\left(\mathrm{t}-2 \tau_{s}\right)^{2}\right), \\
\mathrm{S}\left(\tau_{s}\right)=(\Delta / \gamma)^{2} /[\gamma \mathrm{t}-1+\exp (-\gamma \mathrm{t})] .
\end{gathered}
$$

Here, $\tau_{s}$ is the pulse interval, $\Delta_{i}$ is the inhomogeneous spectral width, $\Delta$ is the coupling strength, and $\gamma$ is the spectral width of the coupling function. The fitting curves are also displayed in Figs. 1 (c) and 2, and very good agreement was obtained. The initial non-exponential decay arises from the dynamical evolution of the wave packet inside dots, and cannot be described by the simple rate equation that gives the exponential decay. This is called in general the non-Markovian relaxation phenomena associated with the transient nonlinear optical effects [5]. It should be noted that the present experiments reveal the non-Markovian nature of the tunneling for the first time. In order to see the electric bias dependence, we have directly solved the timedependent Schrödinger equation. With increasing the magnitude of the electric bias, the echo decay becomes faster. This is because the potential barrier becomes narrow, and the coupling due to the tunneling increases with the bias. The time region of the non-exponential decay also becomes short with increasing the bias, because the initial dynamical motion of the wave packet is suppressed by the strong coupling between the discrete state and the continuum.

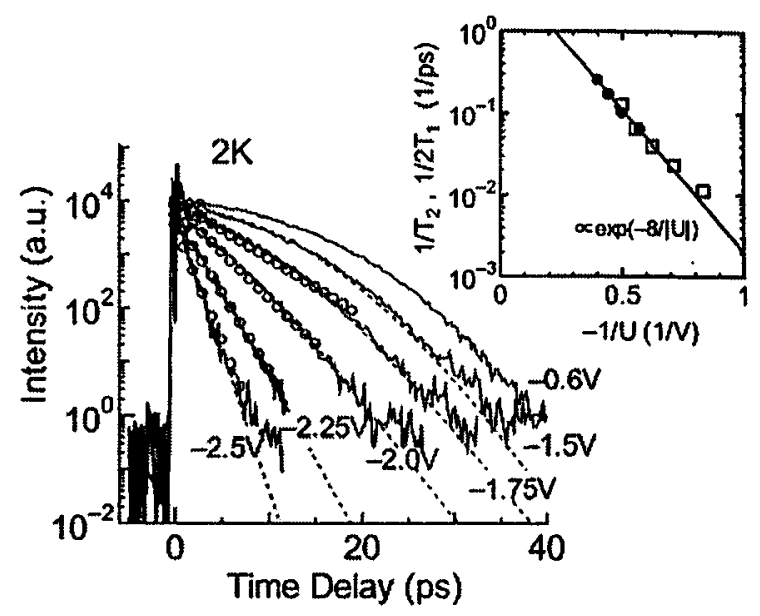

FIGURE 2. Photon echo intensity as a function of electric voltage ranging from $-0.6 \mathrm{~V}$ to $-2.5 \mathrm{~V}$. Dashed lines are the fittings by the non-Markovian theory and open circles are fittings by the exponential decay. An upper figure shows the dephasing rate $(\bullet)$ and half of photoluminescence decay rate (D) obtained by the exponential fitting as a function of inverse of electric bias.

\section{REFERENCES}

1. Yugova, I.A., Gerlovin, I.Ya., Davydov, V.G., Ignatiev, I.V., Kozin, I.E., Ren, H.W., Sugisaki, M., Sugou, S., and Masumoto, Y., Phys. Rev. B 66, 235312-1-9 (2002).

2. Kozin, I.E., Davydov, V.G., Ignatiev, I.V., Kavokin, A.V., Kavokin, K.V., Malpuech, G., Ren, H.-W., Sugisaki, M., Sugou, S., and Masumoto, Y., Phys. Rev. B 65, 241312-1-4(R) (2002).

3. Ignatiev, I.V., Kozin, I.E., Nair, S.V., Ren, H.-W., Sugou, S., and Masumoto, Y., Phys. Rev. B 61, 15633$15636(2000)$.

4. Ignatiev, I.V., Kozin, I.E., Davydov, V.G., Nair, S.V., Lee, J.-S., Ren, H.-W., Sugou, S., and Masumoto, Y., Phys. Rev. B 63, 75316-1-11 (2001).

5. Aihara, M., Phys. Rev. B 25, 53-60 (1982). 